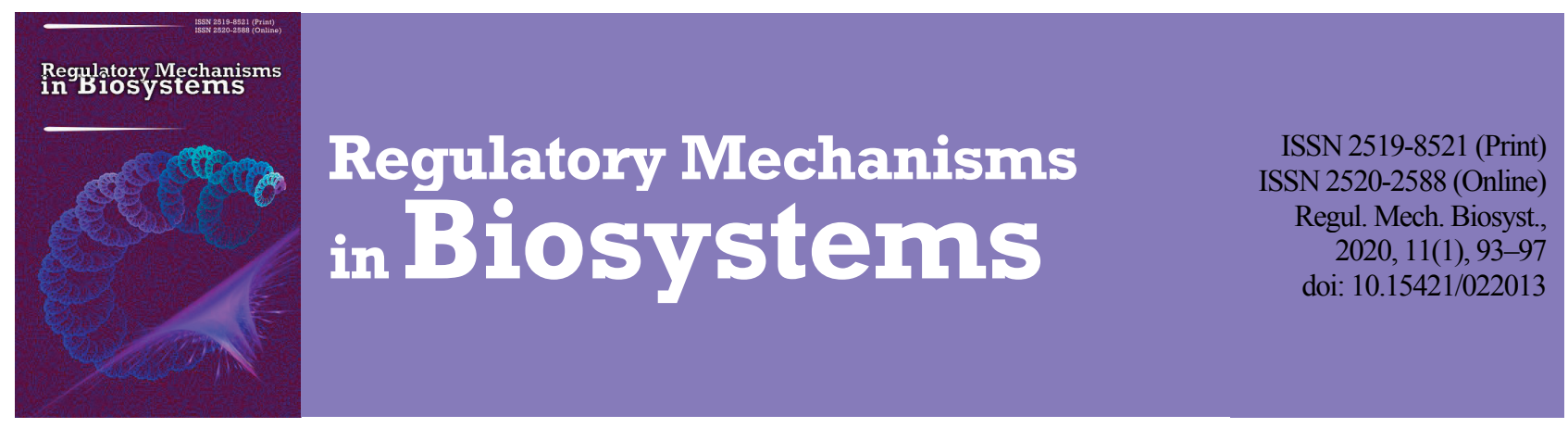

\title{
In vitro immunomodulatory effect of Bifidobacterium bifidum and Lactobacillus reuteri cell free extracts
}

\author{
O. V. Knysh*, M. S. Pogorila*, Y. V. Voyda** \\ *I. I. Mechnikov Institute of Microbiology and Immunology of National Academy of Medical Sciences of Ukraine, Kharkiv, Ukraine \\ **Kharkiv Medical Academy of Postgraduate Education, Kharkiv, Ukraine
}

Article info

Received 17.01.2020 Received in revised form 12.02 .2020

Accepted 13.04.2020

I. I. Mechnikov Institute of Microbiology and Immunology of National Academy of Medical Sciences of Ukraine

Pushkinska st., 14/16,

Kharkiv, 61057, Ukraine.

Kharkiv Medical Academy of Postgraduate Education, Amosova st., 58,

Kharkiv, 61176, Ukraine.

Tel.: +38-066-199-34-72.

E-mail:

knyshoksana@ukr.net
Knysh, O. V., Pogorila, M. S., \& Voyda, Y. V. (2020). In vitro immunomodulatory effect of Bifidobacterium bifidum and Lactobacillus reuteri cell free extracts. Regulatory Mechanisms in Biosystems, 11(1), 93-97. doi:10.15421/022013

Recent studies have shown that alterations of the immune response in the gastrointestinal mucosa are key components of the mechanism of the probiotic action of beneficial bacteria. Most of the beneficial effects of probiotics are due to the action of their structural components and metabolites. Macrophages are first-line defense cells of the immune system, which not only participate in the detection, phagocytosis and destruction of harmful microorganisms, but also determine the nature of the subsequent immune response by presenting antigens to T-cells and initiating inflammation by releasing cytokines. We researched the effect of two types of cell-free extracts (CFEs) containing probiotic derivatives (structural components and metabolites of bacteria) Bifidobacterium bifidum 1 (BbCFE) and Lactobacillus reuteri DSM 17938 (LrCFE) on the activity of mouse peritoneal macrophages and on the ability of peripheral human blood mononuclear cells to produce cytokines. CFEs were obtained by culturing probiotics in their own disintegrates and then removing cells and cell debris by centrifugation and filtration. Peritoneal macrophages were isolated from mice. Some of them were infected in vitro by Salmonella thyphimurium. Uninfected and infected macrophages were incubated in culture medium containing $\left(30 \%\right.$ vol) or not containing CFEs at $37^{\circ} \mathrm{C}$ in a microaerobic atmosphere $\left(5 \% \mathrm{CO}_{2}\right)$ for 18 hours. After incubation, peritoneal macrophages were lysed. The obtained suspensions were centrifuged and supernatants were carefully collected. Macrophages activity was assessed by the nitrites level, superoxide dismutase (SOD), lactate dehydrogenase (LDH) activity and antiinflammatory cytokines levels in supernatants using spectrophotometric method. Peripheral mononuclear cells were isolated from the blood of healthy volunteers. The ability of peripheral mononuclear blood cells to produce antiinflammatory cytokines was evaluated after cell stimulation with lipopolysaccharide (LPS) and incubation with or without CFEs. Cytokine levels in supernatants were determined using enzyme-linked immunosorbent assay (ELISA). After infection with S. thyphimurium in macrophages, nitrite levels increased 5.5-fold, SOD activity 4.8-fold, and LDH 2-fold. Both studied CFEs exerted a similar effect on the macrophages' activity. Addition of BbCFE to the incubation medium of infected macrophages resulted in a 4-fold decrease in nitrite levels, and the addition of LrCFE was accompanied by a decrease in nitrite levels to levels in intact cells. Under the influence of both CFEs, the activity of SOD and LDH was significantly reduced and did not differ significantly from the activity of these enzymes in intact cells. BbCFE and LrCFE did not have a significant effect on nitrite levels, SOD and LDH activity in intact macrophages. Under the influence of $\mathrm{BbCFE}$, there was a 2-fold decrease in the production of TNF, a 2-fold increase in IL10 production, and a 30\% increase in IL6 production by mononuclear cells. LrCFE caused a decrease in TNF production by $26.7 \%$ and IL6 by $36 \%$, and IL10 by 1.9 times. Thus, the studied CFEs normalized the nitrite levels in peritoneal macrophages infected with S. thyphymurium and infection-induced activation of SOD and LDH enzymes. This demonstrates their ability to modulate oxidative processes in macrophages. In addition, under the influence of the investigated CFEs, there was a decrease in the production of pro-inflammatory cytokines (TNF $\alpha$ and IL-6) and increased production of anti-inflammatory cytokine (IL-10) by human peripheral mononuclear cells. The results of the study indicate the ability of CFEs by influencing the functions of innate immunity cells to restrict the inflammatory response and oxidative stress. Based on this, CFEs can be considered as promising agents for the treatment of inflammatory diseases.

Keywords: probiotic derivatives; peritoneal macrophages; peripheral blood mononuclear cells; innate immunity; cytokines.

\section{Introduction}

Nowadays there are plenty of reviewed evidences of health benefits derived from the consumption of probiotic bacteria such as $L$. reuteri and B. bifidum (Sarkar \& Mandal, 2016; Britton, 2017; Mu et al., 2018). Health benefits include improvement of digestion, synthesis of vitamins and amino acids, prevention of gastrointestinal infections by competitive exclusion of pathogens, normalization of carbohydrate and fat metabolism, antitumour effects, improvement of inflammatory bowel disease, adjustment of brain-gut communication, promoting the maturation of the immune system and regulation of its activity (Kristensen et al., 2016; O'Callaghan \& van Sinderen, 2016; Zitvogel et al., 2017; Kysera et al., 2018). Recent studies have shown that alterations of the immune response in the gastrointestinal mucosa are key components of the mechanism of probiotic action of the "health-promoting" bacteria. Probiotics influence the host's immune protection through regulation, stimulation and modulation of immune responses (Azad et al., 2018). The ability of probiotics to enhance the immunological barrier by affecting the innate and adaptive immunity has been reported. But the mechanisms by which they affect immune function are currently not well understood.

Beneficial immunotropic effects of the B. bifidum administration have been shown in the treatment and prevention of upper respiratory tract infections, Clostridium difficile infections, eczema, radiation-related and rotavirus-related diarrhea (Quigley, 2017). B. bifidum shows a dose dependent regulation of Th1/Th2-cytokine levels in mitogen-sti- 
mulated human peripheral blood mononuclear cells (PBMCs). Some members of the $B$. bifidum species can modulate immune responses in vitro by induction of IL-10 production and reducing secretion of TNF- $\alpha$ and IFN- $\gamma$ (Sarkar \& Mandal, 2016; O'Neill et al., 2017). Experimental studies in vivo using model rats have shown that $B$. bifidum OLB6378 modulates mucosal immunity by decreased expression of IL6 and TNF $\alpha$ and improved ileal tight junction (TJ) protein distribution (O'Neill et al., 2017). L. reuteri DSM 17938 has a mild pro-inflammatory effect in vitro and strong anti-inflammatory effect in vivo. It modulates pro-inflammatory signaling via Toll-like receptor 4 (TLR4) and nuclear factor-B (NF-B) which leads to a decrease in pro-inflammatory cytokines of the mucous membrane, such as tumour necrosis factor (TNF)- $\alpha$ and interleukin (IL)-1 $\beta$. L. reuteri modulates the composition of intestinal immune cells in the neonatal gut (dendritic cells (DCs), effector memory T (Tem) cells (eg. TH1/TH2/TH17 cells), and regulatory T (Treg) cells (Hoang et al., 2019). L. reuteri showed the ability to enhance macrophage phagocytosis and increase the expression of interferon (IFN)- $\gamma$, interleukin (IL)-1 $\beta$, IL-12p40, IL-10, chemokine (CXCLi2) and costimulatory molecules (CD40, CD80 and CD86) in macrophages (Taha-Abdelaziz et al., 2019). L. reuteri CRL1324 has demonstrated a protective effect against colonization of Group B Streptococcus by modulating the immune response. The immunomodulatory effect of the $L$. reuteri was to reduce the number of neutrophils induced by the pathogen, to increase the population of activated macrophages, B-lymphocytes and subclasses of IgA and IgG (De Gregorio et al., 2016). L. reuteri has the ability to increase free secretory $\operatorname{IgA}(\operatorname{sgA})$ levels in rats (Wang et al., 2016). Data on the ability of $L$. reuteri to increase salivary IgA levels in patients who use chewing gum containing this probiotic bacterium are contradictory. This, most likely, is due to the difference in the properties of the strains (Mu et al., 2018).

Most of the beneficial effects of probiotics are due to the action of their structural components and metabolites. Microbial structures and metabolites affect the host's immune system by interacting with pattern recognition receptors (PRRs) of the host cells (Levy et al., 2017). Moreover, microbial metabolites can exert not only receptor-mediated, but also direct effect on the metabolism of immune cells (Blacher et al., 2017). According to modern view, bacterial metabolites are messengers between the microbiota and the immune system. Bifidobacterium and Lactobacillus can produce a number of metabolites that modulate the host's immune system activity: short-chain fatty acids (SCFAs), vitamins, amino acids and their derivatives, etc. (Levy et al., 2016). This has been demonstrated by a number of studies. For instance, not $L$. reuteri DSM 12246 cells themselves, but their conditioned supernatants suppressed production of pro-inflammatory cytokines by marrow-derived dendritic cells (Britton, 2017). A culture supernatant of $L$. renteri BM36301 showed the ability to reduce the production of TNF from myeloid cells of human THP-1 (Lee et al., 2016). The histamine which L. reuteri 6475 produces from histidine using the enzyme histidine decarboxylase is able to reduce TNF production by activated monocytes. Although in the context of allergic reactions, histamine is considered as a pro-inflammatory cytokine, studies have shown its anti-inflammatory role in the intestinal tract (Hemarajata et al., 2013; Gao et al., 2015). A number of immunomodulatory effects of $L$. reuteri are associated with the production of tryptophan derivatives, which are recognized as ligands for the aryl hydrocarbon receptor (AhR) (Cervantes-Barragan et al., 2017). EPS from $L$. reuteri induce the anti-inflammatory cytokine production from the macrophages, suppress gene expression of pro-inflammatory cytokines IL-1 $\beta$ and IL-6, induced by $E$. coli infection and induce Foxp3+ regulatory T (Treg) cells in the spleen (Mu et al., 2018; Singh et al., 2018). Some immunomodulatory compounds produced by $L$. reuteri are known, others remains to be discovered. Structural components (cell surface polysaccharides, pili and outer membrane vesicles) and metabolites (SCFAs) of B. bifidum are also able to modulate the immune response (O'Neill et al., 2017; Verma et al., 2018).

The main problem with oral application of cell probiotics is their low survival rate in the gastrointestinal tract. Significant losses of probiotic bacteria may occur during transit through the digestive canal. The main causes of losses are the damaging effects of the gastrointestinal tract chemical factors, low adhesive and colonization ability of the in- troduced probiotics and specific colonization resistance to introduced probiotics in patients (González-Rodríguez et al., 2013; Zmora et al., 2018). In this case, there is no effective interaction of probiotic bacteria with the host immune system. Application of biotechnological products containing probiotic derivatives allow us to avoid the above mentioned and other serious shortcomings and undesirable effects of cell probiotic therapy and quickly achieve the desired effect (Patel et al., 2015). These advantages of cell-free products prompt further studies of unexplored aspects of the mechanism of action of probiotic derivatives, in particular their immunotropic activity.

The aim of the research was to investigate the functional activity of peritoneal macrophages (intact and infected by S. typhymurium) and human peripheral blood mononuclear cells under the influence of cell-free extracts derived from $L$. reuteri (LrCFE) and B. bifidum 1 (BbCFE).

\section{Materials and methods}

Probiotic strains B. bifidum 1 (from dietary supplement "Bifidumbacterin-Biopharma", JSC "Biopharma", Ukraine) and L. reuteri DSM 17938 (from dietary supplement "BioGaia", BioGaia AB, Sweden) were used for obtaining of cell-free extracts (BbCFE and LrCFE). The procedure for obtaining CFEs was described in detail earlier (Knysh et al., 2018). Briefly, bifidobacteria and lactobacilli were cultured for 72 hours in their own disintegrates, obtained by tenfold thermal cycling. The resulting cultures were centrifuged and filtered to remove cells and cell debris.

lisolation of peritoneal macrophages was performed according to the procedure described by Meltzer et al. (1976). To enhance the amount of peritoneal macrophages, white mice were injected with $2.5 \mathrm{~mL}$ per mouse of thioglycollate broth during 4 days. Macrophages were obtained via intraperitoneal injection and then washed with RPMI 1640 at $400 \mathrm{~g}$ for $10 \mathrm{~min}$. Cell viability was tested using trypan blue $(0.2 \%)$ staining.

Human peripheral blood mononuclear cell preparation was performed as described previously (Grievink et al., 2016). Peripheral blood mononuclear cells (PBMC) were obtained from peripheral blood of 9 healthy volunteers. The samples of blood were centrifuged with density gradient $(1.077 \mathrm{~g} / \mathrm{mL}$ ). Pellets were resuspended in RPMI 1640 (Life Technologies, UK) with fetal bovine serum $10 \%$ (inactivated), gentamicine $(50 \mu \mathrm{g} / \mathrm{mL})$, streptomicine $1 \%$, sodium pyruvate solution $(0.23 \mathrm{mmol} / \mathrm{L})$. PBMC were cultured in complete medium $\left(1 \times 10^{6}\right.$ cells $/ \mathrm{mL}$ per well in 96-well plates) in an atmosphere containing $5 \% \mathrm{CO}_{2}$ at $37^{\circ} \mathrm{C}$.

Cultivation of peripheral blood mononuclear cells in presence of $C F E$. The cell-free extracts from B. bifidum $(\mathrm{BbCFE})$ and $L$. reuteri (LrCFE) were added to PBMC $\left(1 \times 10^{6}\right.$ cells $/ \mathrm{mL}$ per well $)$ and incubated for 72 hours in an atmosphere containing $5 \% \mathrm{CO}_{2}$ at $37^{\circ} \mathrm{C}$. The concentration of CFEs in the incubation medium was $30 \%$ vol. Lipopolysaccharide (LPS) (Sigma, USA) in concentration $20 \mathrm{ng} / \mathrm{mL}$ was used in this in vitro experiment as a control of stimulation of cytokines production by PBMC. After incubation, supernatants were collected, centrifuged and stored $\left(-20^{\circ} \mathrm{C}\right)$ for further measuring of cytokine levels.

Cytokine levels measurement in supernatant of cultivated peripheral blood mononuclear cells in presence of CFEs by enzyme-linked immunosorbent assay (ELISA). Appropriate commercial ELISA kits were used to measure TNF $\alpha$ (TNF alpha Human ELISA Kit, 96 tests, Invitrogen, USA), assay range $15-1500 \mathrm{pg} / \mathrm{mL}$ (analytical sensitivity $<$ $3 \mathrm{pg} / \mathrm{mL}$, Interassay CV - 6\%), IL-6 (IL-6 Human ELISA Kit, 96 tests, Invitrogen, USA), assay range $7.8-2500 \mathrm{pg} / \mathrm{mL}$ (Analytical sensitivity $<2 \mathrm{pg} / \mathrm{mL}$, Interassay CV - 7.9\%), and IL-10 (IL-10 Human ELISA Kit, 96 tests, Invitrogen, USA), assay range $11-1335 \mathrm{pg} / \mathrm{mL}$ (analytical sensitivity $<1 \mathrm{pg} / \mathrm{mL}$, Interassay $\mathrm{CV}-2.8 \%$ ). For measurement of optical density values of samples, we used plate reader Stat-Fax 3000+ (USA) at $490 \mathrm{mn}$.

Infection of isolated peritoneal macrophages by Salmonella thyphimurium in presence of CFEs. For observation of the capacity of CFEs to modulate macrophage function, peritoneal macrophages isolated from mice were infected in vitro by S. thyphimurium (Qureshi et al., 1996). Peritoneal macrophages $\left(10^{6} \mathrm{~mL}^{-1}\right)$ were incubated with bacterial suspension of $S$. thyphimurium $\left(\sim 10^{8} \mathrm{CFU} / \mathrm{mL}\right)(50: 1)$ for 5 hours. Then, infected macrophages of the first group $(n=11)$ were incubated in the 
presence of BbCFE (30\% vol), infected macrophages of the second group $(\mathrm{n}=11)$ were incubated in the presence of LrCFE $(30 \% \mathrm{vol})$ and infected macrophages of the third group $(n=11)$ were incubated without CFEs at $37{ }^{\circ} \mathrm{C}$ in an atmosphere containing $5 \% \mathrm{CO}_{2}$ for 18 hours. After incubation, all groups of peritoneal macrophages were lysed using lysis buffer $(1: 1)$ during $20 \mathrm{~min}$ at $4{ }^{\circ} \mathrm{C}$. Then the obtained suspensions were centrifuged at $300 \mathrm{~g}$ for $15 \mathrm{~min}$. Carefully collected supernatant was used for measuring the several parameters which characterize macrophages function.

Nitrite levels were measured in the supernatant of macrophages' culture using the method described by Green with coworkers (Green et al., 1982). The absorbance was measured at $546 \mathrm{~nm}$. The quantity of nitrite was determined using sodium nitrite standard graph.

Superoxide dismutase activity (SOD) was measured in the supernatant of cell culture using the method described by Kono (1978). The absorbance was read at $560 \mathrm{~nm}$ for $3 \mathrm{~min}$ with $0.5 \mathrm{~min}$ interval. SOD activity was expressed in units of SOD per $\mathrm{mg}$ of protein (the one unit activity is the amount of SOD, which is required to inhibit the rate of reduction of nitroblue tetrasolium (NBT) by $50 \%$ ). The protein level in supernatant of cell culture was measured using the method described by Lowry et al. (1951).

Lactate dehydrogenase activity (LDH) was measured in the supernatant of cell culture using the method described by Bergmeyer \& Bernt (1974). The absorbance was measured at $340 \mathrm{~nm}$.

Obtained data were expressed as mean arithmetic value with standard deviation $(\mathrm{x} \pm \mathrm{SD})$. Statistical analysis was performed by ANOVA, followed by post hoc Fisher's test for comparison with the control samples. A 95\% confidence interval was considered.

\section{Results}

Nitrite levels in macrophages infected with S. thyphimurium increased 5.5-fold compared to nitrite levels measured in intact macrophages $(6.28 \pm 0.51$ vs $1.14 \pm 0.12 \mu \mathrm{g} / \mathrm{mL}, \mathrm{P}<0.05)$. The addition to the culture medium of $\mathrm{BbCFE}$ led to a significant decrease of nitrite levels in infected macrophages. The specified parameter was 4 times lower than in infected macrophages incubated without BbCFE (1.54 \pm 0.14 vs $6.28 \pm 0.51 \mu \mathrm{g} / \mathrm{mL}, \mathrm{P}<0.05)$. But nitrite levels in infected macrophages under the influence of BbCFE didn't reach initial values $(1.54 \pm 0.14$ vs $1.14 \pm 0.12 \mu \mathrm{g} / \mathrm{mL}, \mathrm{P}<0.05)$. Incubation of infected macrophages with LrCFE led to recovery of their nitrite levels to initial values $(1.29 \pm 0.12$ vs $1.14 \pm 0.12 \mu \mathrm{g} / \mathrm{mL}$, no statistical differences, $\mathrm{P}>$ 0.05). Both studied CFEs did not significantly change the nitrite levels in intact macrophages.

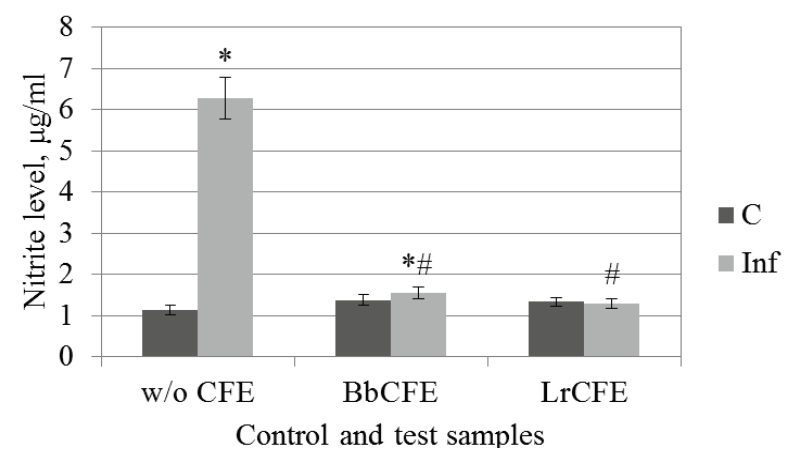

Fig. 1. The effect of BbCFE and LrCFE on the nitrite levels in macrophages ( $\mathrm{x} \pm \mathrm{SD}$ at $546 \mathrm{~nm}, \mathrm{n}=11)$ : $\mathrm{C}$ - control samples (intact macrophages); Inf - test samples (infected macrophages); w/o CFE incubated without CFE; BbCFE - incubated with BbCFE; LrCFE incubated with LrCFE; the differences are significant compared to the:

* - control sample w/o CFE; ${ }^{*}$ - test sample w/o CFE; $\mathrm{P}<0.05$

SOD activity determined in infected macrophages was elevated 4.8-fold compared to SOD activity in intact macrophages $(6.38 \pm 0.58$ vs $1.34 \pm 0.21$ units $/ \mathrm{mg}$ protein, $\mathrm{P}<0.05$ ). The addition of $\mathrm{BbCFE}$ and LrCFE to the culture medium reduced the SOD activity in infected macrophages to the initial levels $(1.34 \pm 0.21$ vs $1.43 \pm 0.21$ and $1.60 \pm$
0.18 units $/ \mathrm{mg}$ protein, $\mathrm{P}>0.05$ ). SOD activity in infected macrophages incubated with BbCFE was 4.5 times lower than SOD activity in infected macrophages incubated without extracts $(1.43 \pm 0.21$ vs $6.38 \pm 0.58$ units/mg protein, $\mathrm{P}<0.05$ ). The addition of LrCFE to the incubation medium led to a 4-fold decrease in SOD activity in infected macrophages $(1.60 \pm 0.18$ vs $6.38 \pm 0.58, P<0.05)$. Incubation of intact macrophages with CFEs did not change the SOD activity since the studied parameter remained at the initial level $(1.34 \pm 0.21$ vs $1.27 \pm 0.17$ and $1.16 \pm 0.19$ units/mg protein, no statistical differences, $\mathrm{P}>0.05$ ).

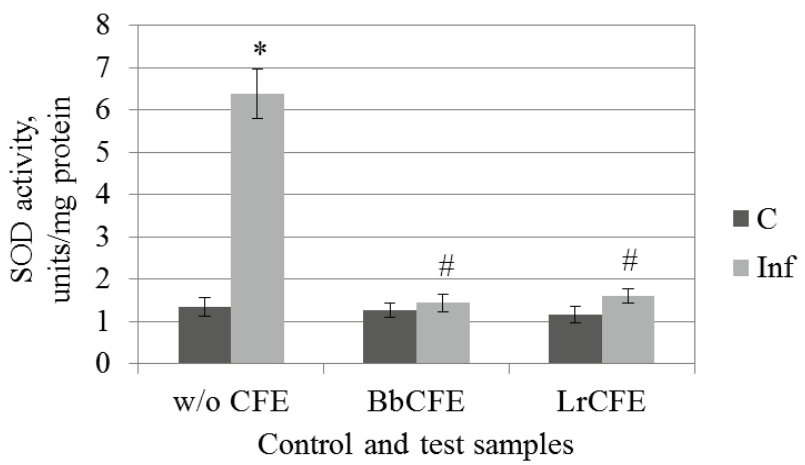

Fig. 2. The effect of BbCFE and LrCFE on the SOD activity in macrophages ( $\mathrm{x} \pm \mathrm{SD}$ at $560 \mathrm{~nm}, \mathrm{n}=11)$ : $\mathrm{C}$ - control samples (intact macrophages); Inf - test samples (infected macrophages); w/o CFE incubated without CFE; BbCFE - incubated with BbCFE; LrCFE incubated with LrCFE; the differences are significant compared to the:

* - control sample w/o CFE; ${ }^{\#}$ - test sample w/o CFE; $\mathrm{P}<0.05$

The 2-fold elevation of LDH activity was observed in infected macrophages compared to LDH activity in intact cells $(42.51 \pm 3.92$ vs $21.10 \pm 1.78 \mathrm{mmol} \mathrm{NADH} / \mathrm{min} / \mathrm{mg}$ protein, $\mathrm{P}<0.05$ ). Incubation of infected macrophages with BbCFE led to a decrease of LDH activity in infected macrophages to the initial level $(20.13 \pm 2.04$ vs $21.10 \pm 1.78$ $\mathrm{mmol} \mathrm{NADH} / \mathrm{min} / \mathrm{mg}$ protein, $\mathrm{P}>0.05$ ). $\mathrm{LDH}$ activity under the influence of BbCFE was 2.1 times lower than in the case of incubation of infected macrophages without the indicated extract $(20.13 \pm 2.04$ vs $42.51 \pm 3.92 \mathrm{mmol} \mathrm{NADH} / \mathrm{min} / \mathrm{mg}$ protein, $\mathrm{P}<0.05$ ). As a result of the introduction of LrCFE into the incubation medium, a 2.2-fold decrease of the LDH activity in infected macrophages was observed (19.0 \pm 1.81 vs $42.51 \pm 3.92 \mathrm{mmol} \mathrm{NADH} / \mathrm{min} / \mathrm{mg}$ protein, $\mathrm{P}<0.05$ ). The $\mathrm{LDH}$ activity was at the level of enzyme activity in intact macrophages $(19.0 \pm 1.81$ vs $21.10 \pm 1.78, \mathrm{P}>0.05)$. The addition of both CFEs did not affect the LDH activity of intact macrophages $(21.10 \pm 1.78$ vs $20.13 \pm 2.04$ and $19.0 \pm 1.81, \mathrm{P}>0.05$ ).

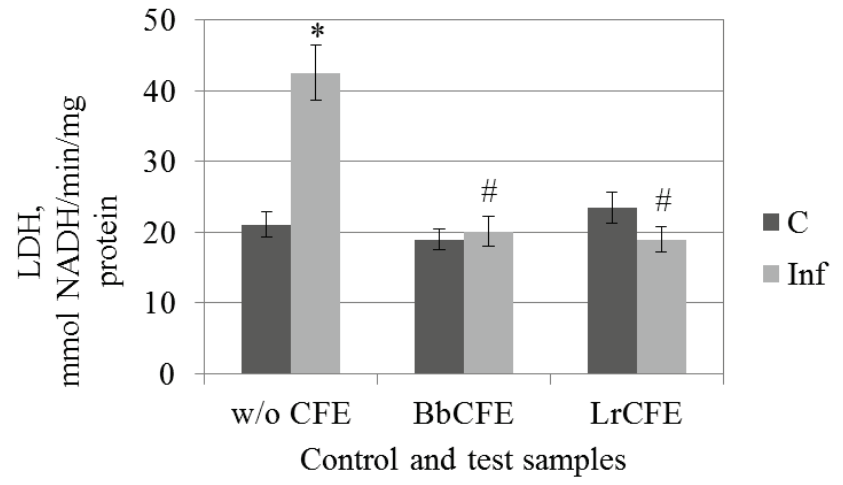

Fig. 3. The effect of BbCFE and LrCFE on the LDH activity in macrophages ( $\mathrm{x} \pm \mathrm{SD}$ at $340 \mathrm{~nm}, \mathrm{n}=11): \mathrm{C}$ - control samples (intact macrophages); Inf - test samples (infected macrophages); w/o CFE - incu-

bated without CFE; BbCFE - incubated with BbCFE; LrCFE incubated with LrCFE; the differences are significant compared to the:

* - control sample w/o CFE; ${ }^{*}$ - test sample w/o CFE; $\mathrm{P}<0.05$

As can be seen from the data presented in Table 1, BbCFE and LrCFE differently affect the production of pro- and anti-inflammatory 
cytokines by PBMC. Under the influence of BbCFE, a 2-fold decrease in the level of TNF $\alpha$ was observed compared to the LPS-induced level of TNF $\alpha$ after 24 hours of incubation $(\mathrm{P}<0.05)$. Regarding the production of pro-inflammatory cytokine IL-6, the addition of BbCFE to the PBMC culture medium led to a statistically significant increase in its production, although to a small extent $(\mathrm{P}<0.05)$. A 2-fold increase in the production of the anti-inflammatory cytokine IL-10 was observed in this test sample $(\mathrm{P}<0.05)$. Under the influence of LrCFE, the level of TNF $\alpha$ production decreased by $26.7 \%$ compared with the level in the control sample (induced by LPS, $\mathrm{P}<0.05$ ). Due to the addition of LrCFE to the PBMC culture medium, the level of pro-inflammatory cytokine IL-6 production decreased by $36 \%(\mathrm{P}<0.05)$. As a result of adding LrCFE to the PBMC culture medium, we obtained a 1.9-fold increase in the production of anti-inflammatory cytokine IL-10 compared to the LPS control sample (induced by LPS, $\mathrm{P}<0.05$ ).

\section{Table 1}

The effect of BbCFE and LrCFE on the cytokine levels in supernatant of $\mathrm{PBMC}(\mathrm{x} \pm \mathrm{SD}, \mathrm{n}=11)$

\begin{tabular}{lccc}
\hline \multirow{2}{*}{ Samples } & \multicolumn{3}{c}{ Cytokine levels, $\mathrm{x} \pm \mathrm{SD}$} \\
\cline { 2 - 4 } & $\mathrm{TNF} \alpha, \mathrm{pg} / \mathrm{mL}$ & $\mathrm{IL}-6, \mathrm{pg} / \mathrm{mL}$ & $\mathrm{IL}-10, \mathrm{pg} / \mathrm{mL}$ \\
\hline Control & $1008 \pm 76$ & $25.0 \pm 1.3$ & $1256 \pm 100$ \\
BbCFE & $567 \pm 105^{*}$ & $30.7 \pm 1.4^{*}$ & $2595 \pm 150^{*}$ \\
LrCFE & $739 \pm 60^{*}$ & $16.1 \pm 1.1^{*}$ & $2387 \pm 46^{*}$ \\
\hline
\end{tabular}

Note: $\mathrm{C}$ - control samples (with application of LPS); BbCFE - incubated with BbCFE; LrCFE - incubated with LrCFE; the differences are significant compared to the: * - control sample, $\mathrm{P}<0.05$.

\section{Discussion}

CFEs of probiotic origin are known to contain a number of soluble factors: metabolites and cytoplasmic components that are capable of exerting an antimicrobial effect and are active in relation to cell functionality, including hydrogen peroxide, lactic acid, reuterin, DNA (CpG motifs) and other molecules (Garbi et al., 2004; Singh et al., 2018). Antimicrobial factors aimed at inhibiting ligand-receptor interplay between pathogenic bacteria and enterocytes alter epithelial permeability. Full benefits will be obtained, if direct antibacterial action is accompanied by the capacity to enhance the mucosal immunity against infectious agents provided by macrophages via enhancing the enzyme activity in presence of pathogen and modulating immune-regulating cytokines. It is considered that these soluble factors and various cytoplasmic bacterial constituents are involved in the regulation of cytokine induction (Blacher et al., 2017).

Besides phagocytosis, nitric oxide production is another option that could indicate macrophage activation. As is known, one of the major functions of nitric oxide (NO) is the participation in antimicrobial innate defense. This is an important signaling molecule that acts in different tissues. It is involved in the pathogenesis and control of infectious diseases via its variety of reaction partners (DNA, proteins, low-molecular weight thiols, prosthetic groups, reactive oxygen intermediates). NO's activity is strongly influenced by its concentration. The effects of protection against pathogenic microorganisms and toxic effects of $\mathrm{NO}$ on tissues are often observed in parallel. In our study, we showed an increase in nitrite levels in infected macrophages, which was supplemented by an increase in TNF $\alpha$ and IL-6 levels. Normalization of nitrite levels in infected macrophages in the presence of CFEs is considered as their ability to influence NO-producing signaling pathways that pass through pro-inflammatory interleukins (Soufli et al., 2016).

The SOD family plays an important role in oxidative stress modulating (Bresciani et al., 2015). The infection process is always accompanied by oxidative stress through increased production of multiple reactive oxygen and nitrogen species. Decreasing of SOD activity in supernatant of cultivated macrophages infected with $S$. thyphimurium is considered as capacity of CFEs to eliminate some reactive molecules with decreasing of oxidative stress.

The intracellular lactate metabolism is modulated during inflammatory activation of innate immune cells. Furthermore, extracellular lactate affects immune cell metabolism and cytokine production depending on time and concentration and, thus, can serve as a negative feedback signal limiting inflammation. We consider the decrease in LDH activity in the supernatant of cultured infected macrophages as the ability of CFEs to restrict the chain reaction of pro-inflammatory activity, which can play a positive role in limiting acute infectious inflammation. In general, additional studies are needed, in particular, the measurement of cytokines and oxidative stress in cells of innate immunity.

Several clinical studies show that gut inflammation is accompanied by maintained pro-inflammatory response. Cytokines are key regulators of inflammation. Several pro-inflammatory and immune regulatory cytokines are dysregulated in the mucosa in inflammation disease. Some clinical results in patients with acute inflammatory bowel disease suggest that the use of probiotics can modulate the pro-inflammatory response, improving the barrier functions of the mucous membrane (Wasilewski et al., 2015; Zhang et al., 2017; Yu et al., 2019).

$\mathrm{TNF} \alpha$ is a pro-inflammatory cytokine released during a bacterial infection, along with other pro-inflammatory cytokines, such as IL-1, IL-6. $\mathrm{TNF} \alpha$ is known as a stimulant of iNOS activity, which can lead to increased levels of NO. TNF $\alpha$ plays a key role in destruction of tissue during inflammation, thus it is involved in epithelial damage in inflammatory bowel disease. In our study we observed a significant increase of TNF $\alpha$ level in macrophages infected with S. thyphimurium.

IL-6 is an interleukin that acts as both a pro-inflammatory cytokine and an anti-inflammatory myokine. The role of IL-6 as an anti-inflammatory myokine is mediated through its inhibitory effects on TNF $\alpha$ and IL-1, and activation of IL-1ra and IL-10. Thus, production of IL-6 may be induced as a reaction to elevation of TNF $\alpha$ synthesis. So, we could see also an increase in the IL-6 level in infected macrophages.

IL-10 also known as human cytokine synthesis inhibitory factor is an anti-inflammatory cytokine. IL-10 has multiple, pleiotropic, effects in immunoregulation and inflammation. This cytokine downregulates the production of Th1 cytokines and the expression of co-stimulatory molecules on macrophages. IL-10 can block NF- $\mathrm{KB}$ activity, and is involved in the regulation of the JAK-STAT signaling pathway. It is shown that IL-10 predominantly inhibits LPS and bacterial product mediated induction of the pro-inflammatory cytokines TNF $\alpha$, IL-1 $\beta$, IL-12 and IFN $\gamma$ secretion.

In relation to production of cytokines, the implication of $\mathrm{BbCFE}$ to peripheral blood mononuclear cells led to enhancing of IL-10 production and decreasing of TNF $\alpha$ and IL- 6 synthesis. The application of LrCFE shows a similar effect: a decrease in TNF $\alpha$ and IL-6 levels and an increase in IL-10 levels. The difference of enhancing and decreasing of pro- and anti-inflammatory immune-regulatory cytokines can be explained by the distinction of metabolic and structural constituents of $\mathrm{BbCFE}$ and LrCFE. Some articles attribute this difference to the exertion of the more pronounced modulatory effect by some $\mathrm{CpG}$ motives compared with other motives (Garbi et al., 2004). It is suggested that high CpG content presented in BbCFE may account for the high cytokine response, in particular IL-10. Especially the significant role in modulating of pro- and anti-inflammatory cytokines refer to bacterial DNA containing unmethylated $\mathrm{CpG}$ motifs, which is a pathogen-associated molecular pattern and interacts with host immune cells via a TLR inducing cytokines production. This study gives a clearer understanding of CFEs' influence at modulation of immune-regulating cytokines and opens the perspectives for implication of cell-free extracts based on probiotics to treat the inflammatory disease via their ability to effect the function of macrophages and mononuclear blood cells, which are the major link of antimicrobial defense.

\section{Conclusion}

The obtained results show that CFEs derived from $L$. reuteri and B. bifidum are capable of normalizing the nitrite levels in peritoneal macrophages infected by $S$. thyphymurium. The detected ability of BbCFE and LrCFE to normalize the infection-induced activation of SOD and LDH enzymes indicates their ability to modulate the oxidative processes in macrophages. Along with this, the studied extracts showed the ability to reduce the production of pro-inflammatory cytokines (TNF $\alpha$ and IL-6) and to increase the production of anti-inflammatory 
cytokine (IL-10) by peripheral human mononuclear blood cells. The results of the study indicate the ability of CFEs, through the influence on the function of cells of innate immunity, to restrict the pro-inflammatory response and oxidative stress. Based on this, CFEs can be considered as promising agents for the treatment of inflammatory diseases.

\section{References}

Azad, M. A. K., Sarker, M., \& Wan, D. (2018). Immunomodulatory effects of probiotics on cytokine profiles. BioMed Research International, 2018, 1-10.

Bergmeyer, H. U., \& Bernt, E. (1974). UV-assay with pyruvate and NADH. In: Methods of enzymatic analysis. Academic Press. Pp. 574-579.

Blacher, E., Levy, M., Tatirovsky, E., \& Elinav, E. (2017). Microbiome-modulated metabolites at the interface of host immunity. The Journal of Immunology, 198(2), 572-580.

Bresciani, G., da Cruz, I. B. M., \& González-Gallego, J. (2015). Manganese superoxide dismutase and oxidative stress modulation. Advances in Clinical Chemistry, 68, 87-130.

Britton, R. A. (2017). Lactobacillus reuteri. In: Floch, M., Ringel, Y., \& Walker, W. (Eds.). The microbiota in gastrointestinal pathophysiology: Implications for human health, prebiotics, probiotics, and dysbiosis. Academic Press, London. Pp. 89-97.

Cervantes-Barragan, L., Chai, J. N., Tianero, M. D., Di Luccia, B., Ahern, P. P., Merriman, J., Cortez, V. S., Caparon, M. G., Donia, M. S., Gilfillan, S., Cella, M., Gordon, J. I., Hsieh, C. S., \& Colonna, M. (2017). Lactobacillus reuteri induces gut intraepithelial CD4+CD8 $\alpha \alpha+T$ cells. Science, 357(6353), 806-810.

De Gregorio, P. R., Juárez Tomás, M. S., \& Nader-Macías, M. E. F. (2015). Immunomodulation of Lactobacillus reuteri CRL1324 on Group B Streptococcus vaginal colonization in a murine experimental model. American Journal of Reproductive Immunology, 75(1), 23-35.

Gao, C., Major, A., Rendon, D., Lugo, M., Jackson, V., Shi, Z., Mori-Akiyama, Y., \& Versalovic, J. (2015). Histamine H2 receptor-mediated suppression of intestinal inflammation by probiotic Lactobacillus reuteri. mBio, 6(6), e01358-15.

Garbi, N., Arnold, B., Gordon, S., Hämmerling, G. J., \& Ganss, R. (2004). CpG motifs as proinflammatory factors render autochthonous tumors permissive for infiltration and destruction. The Journal of Immunology, 172(10), 5861-5869.

González-Rodríguez, I., Ruiz, L., Gueimonde, M., Margolles, A., \& Sánchez, B. (2012). Factors involved in the colonization and survival of bifidobacteria in the gastrointestinal tract. FEMS Microbiology Letters, 340(1), 1-10.

Green, L. C., Wagner, D. A., Glogowski, J., Skipper, P. L., Wishnok, J. S., \& Tannenbaum, S. R. (1982). Analysis of nitrate, nitrite, and $\left[{ }^{15} \mathrm{~N}\right]$ nitrate in biological fluids. Analytical Biochemistry, 126(1), 131-138.

Grievink, H. W., Luisman, T., Kluft, C., Moerland, M., \& Malone, K. E. (2016) Comparison of three isolation techniques for human peripheral blood mononuclear cells: Cell recovery and viability, population composition, and cell functionality. Biopreservation and Biobanking, 14(5), 410-415.

Hemarajata, P., Gao, C., Pflughoeft, K. J., Thomas, C. M., Saulnier, D. M., Spinler, J. K., \& Versalovic, J. (2013). Lactobacillus reuteri-specific immunoregulatory gene rsiR modulates histamine production and immunomodulation by Lactobacillus reuteri. Journal of Bacteriology, 195(24), 5567-5576.

Hoang, T. K., Freeborn, J., Wang, T., Mai, T., He, B., Park, S., Tran, D. Q., Roos, S., Rhoads, J. M., \& Liu, Y. (2019). Human breast milk promotes the immunomodulatory function of probiotic Lactobacillus reuteri DSM 17938 in the neonatal rat intestine. Journal of Probiotics and Health, 7(1), 210.

Knysh, O. V., Isaenko, O. Y., Babych, E. M., Kompaniets, A. M., Pakhomov, O. V., Polyanska, V. P., Zachepylo, S. V., \& Danilova, I. S. (2018). Antimicrobial activity of bifidobacteria derivatives after storage in a frozen state. Problems of Cryobiology and Cryomedicine, 28(3), 237-248.

Kono, Y. (1978). Generation of superoxide radical during autoxidation of hydroxylamine and an assay for superoxide dismutase. Archives of Biochemistry and Biophysics, 186(1), 189-195.

Kristensen, N. B., Bryrup, T., Allin, K. H., Nielsen, T., Hansen, T. H., \& Pedersen, O. (2016). Alterations in fecal microbiota composition by probiotic supplementation in healthy adults: A systematic review of randomized controlled trials. Genome Medicine, 8(1), 52.

Kysera, Y. V., Storchak, Y. G., \& Gutyj, B. V. (2018). Experimental study of immunoprophylactic anti-pneumococcal medicine and its immunogenic properties. Ukrainian Journal of Ecology, 8(1), 307-316.

Lee, J., Yang, W., Hostetler, A., Schultz, N., Suckow, M. A., Stewart, K. L., Kim, D. D., \& Kim, H. S. (2016). Characterization of the anti-inflammatory Lactobacillus reuteri BM36301 and its probiotic benefits on aged mice. BMC Microbiology, 16, 1.
Levy, M., Blacher, E., \& Elinav, E. (2017). Microbiome, metabolites and host immunity. Current Opinion in Microbiology, 35, 8-15.

Levy, M., Thaiss, C. A., \& Elinav, E. (2016). Metabolites: Messengers between the microbiota and the immune system. Genes and Development, 30(14), 1589 1597.

Lowry, O. H., Rosebrough, N. J., Farr, A. L., \& Randall, R. J. (1951). Protein measurement with the Folin phenol reagent. Journal of Biological Chemistry, 193, 265-275.

Meltzer, M. S., Stevenson, M. M., Tucker, R. W., \& Leonard, E. J. (1976). Peritoneal macrophages from BCG-infected mice: Tumor cytotoxicity and chemotactic responses in vitro. In: The macrophage in neoplasia. Academic Press, New York. P. 211.

Mu, Q., Tavella, V. J., \& Luo, X. M. (2018). Role of Lactobacillus reuteri in human health and diseases. Frontiers in Microbiology, 9, 757.

O’Callaghan, A., \& van Sinderen, D. (2016). Bifidobacteria and their role as members of the human gut microbiota. Frontiers in Microbiology, 7, 925.

O’Neill, I., Schofield, Z., \& Hall, L. J. (2017). Exploring the role of the microbiota member Bifidobacterium in modulating immune-linked diseases. Emerging Topics in Life Sciences, 1(4), 333-349.

Patel, R., \& DuPont, H. L. (2015). New approaches for bacteriotherapy: Prebiotics, new-generation probiotics, and synbiotics. Clinical Infectious Diseases, 60(suppl. 2), S108-S121.

Quigley, E. M. M. (2016). Bifidobacterium bifidum. In: Floch, M. H., Ringel, Y., \& Walker, W. A. (Eds.). The microbiota in gastrointestinal pathophysiology: Implications for human health, prebiotics, probiotics, and dysbiosis. Elsevier. Part B, Chaptter 14, 131-133.

Qureshi, T., Templeton, J. W., \& Adams, L. G. (1996). Intracellular survival of Brucella abortus, Mycobacterium bovis BCG, Salmonella dublin, and Salmonella typhimurium in macrophages from cattle genetically resistant to Brucella abortus. Veterinary Immunology and Immunopathology, 50(1-2), 55-65.

Sarkar, A., \& Mandal, S. (2016). Bifidobacteria - Insight into clinical outcomes and mechanisms of its probiotic action. Microbiological Research, 192, 159-171.

Singh, A., Vishwakarma, V., \& Singhal, B. (2018). Metabiotics: The functional metabolic signatures of probiotics: Current state-of-art and future research priorities. Metabiotics: Probiotics effector molecules. Advances in Bioscience and Biotechnology, 9(4), 147-189.

Soufli, I., Toumi, R., Rafa, H., \& Touil-Boukoffa, C. (2016). Overview of cytokines and nitric oxide involvement in immuno-pathogenesis of inflammatory bowel diseases. World Journal of Gastrointestinal Pharmacology and Therapeutics, 7(3), 353 .

Taha-Abdelaziz, K., Astill, J., Kulkarni, R. R., Read, L. R., Najarian, A., Farber, J. M., \& Sharif, S. (2019). In vitro assessment of immunomodulatory and antiCampylobacter activities of probiotic lactobacilli. Scientific Reports, 9, 17903.

Verma, R., Lee, C., Jeun, E.-J., Yi, J., Kim, K. S., Ghosh, A., Byun, S., Lee, C.-G., Kang, H.-J., Kim, G.-C., Jun, C.-D., Jan, G., Suh, C.-H., Jung, J.-Y., Sprent, J., Rudra, D., De Castro, C., Molinaro, A., Surh, C. D., \& Im, S.-H. (2018). Cell surface polysaccharides of Bifidobacterium bifidum induce the generation of Foxp3+ regulatory T cells. Science Immunology, 3(28), eaat6975.

Wang, P., Li, Y., Xiao, H., Shi, Y., Le, G. W., \& Sun, J. (2016). Isolation of Lactobacillus reuteri from Peyer's patches and their effects on sIgA production and gut microbiota diversity. Molecular Nutrition and Food Research, 60(9), 2020-2030.

Wasilewski, A., Zielińska, M., Storr, M., \& Fichna, J. (2015). Beneficial effects of probiotics, prebiotics, synbiotics, and psychobiotics in inflammatory Bowel disease. Inflammatory Bowel Diseases, 21(7), 1674-1682.

Yu, H.-S., Lee, N.-K., Choi, A.-J., Choe, J.-S., Bae, C. H., \& Paik, H.-D. (2019). Anti-inflammatory potential of probiotic strain Weissella cibaria JW15 isolated from kimchi through regulation of NF- $\mathrm{KB}$ and MAPKs pathways in LPS-induced RAW 264.7 cells. Journal of Microbiology and Biotechnology, 29(7), 1022-1032.

Zhang, M., Sun, K., Wu, Y., Yang, Y., Tso, P., \& Wu, Z. (2017). Interactions between intestinal microbiota and host immune response in inflammatory bowel disease. Frontiers in Immunology, 8, 942.

Zitvogel, L., Daillère, R., Roberti, M. P., Routy, B., \& Kroemer, G. (2017). Anticancer effects of the microbiome and its products. Nature Reviews Microbiology, 15(8), 465-478.

Zmora, N., Zilberman-Schapira, G., Suez, J., Mor, U., Dori-Bachash, M., Bashiardes, S., Zur, M., Regev-Lehavi, D., Brik, R. B.-Z., Federici, S., Hom, M., Cohen, Y., Moor, A. E., Zeevi, D., Korem, T., Kotler, E., Harmelin, A., Itzkovitz, S., Maharshak, N., Shibolet, O., Pevsner-Fischer, M., Shapiro, H., Sharon, I., Halpern, Z., Segal, E., \& Elinav, E. (2018). Personalized gut mucosal colonization resistance to empiric probiotics is associated with unique host and microbiome features. Cell, 174(6), 1388-1405. 